\title{
openheart Low myocardial energetic efficiency is associated with increased mortality in aortic stenosis
}

\author{
Edda Bahlmann (10 , ${ }^{1}$ Eigir Einarsen (1) , ${ }^{2}$ Dana Cramariuc, ${ }^{2,3}$ Helga Midtbø, ${ }^{3}$ \\ Costantino Mancusi, ${ }^{4}$ Anne Rossebø, ${ }^{5}$ Stephan Willems, ${ }^{1}$ Eva Gerdts ${ }^{2}$
}

To cite: Bahlmann E, Einarsen $\mathrm{E}$, Cramariuc D, et al. Low myocardial energetic efficiency is associated with increased mortality in aortic stenosis. Open Heart 2021;8:e001720. doi:10.1136/ openhrt-2021-001720

Received 13 May 2021 Accepted 2 August 2021

Q Check for updates

(C) Author(s) (or their employer(s)) 2021. Re-use permitted under CC BY-NC. No commercial re-use. See rights and permissions. Published by BMJ.

'Department of Cardiology, Asklepios Kliniken St. Georg, Hamburg, Germany

${ }^{2}$ Department of Clinical Science, University of Bergen Department of Medicine, Bergen, Norway

${ }^{3}$ Department of Heart Disease, Haukeland University Hospital Department of Heart Disease, Bergen, Norway

${ }^{4}$ Department of Advanced Biomedical Science and Hypertension Research Center, Federico II University of Naples, Naples, Italy

${ }^{5}$ Department of Cardiology, 0slo University Hospital Ullevaal, Oslo, Norway

Correspondence to Dr Edda Bahlmann; doc_edda@ hotmail.com

\section{ABSTRACT}

Objectives In hypertension, low myocardial energetic efficiency (MEEi) has been documented as an integrated marker of metabolic and left ventricular (LV) myocardial dysfunction. We tested the predictive performance of MEEi in initially asymptomatic aortic stenosis (AS) patients free from diabetes and known cardiovascular disease.

Methods Data from 1703 patients with mostly moderate AS enrolled in the Simvastatin and Ezetimibe in Aortic Stenosis study followed for 4.3 years was used. MEE was calculated from Doppler stroke volume/([heart rate/60]) and indexed to LV mass (MEEi). The threshold value for MEEi associated with increased mortality was identified in generalised additive model with smoothing splines. Covariables of MEEi were identified in logistic regression analysis. Outcome was assessed in Cox regression analysis and reported as $\mathrm{HR}$ and $95 \% \mathrm{Cl}$.

Results MEEi $<0.34 \mathrm{~mL} / \mathrm{s}$ per gram was associated with increased cardiovascular mortality $(n=80)$ (HR $2.53(95 \%$ Cl 1.50 to 4.28$))$ and all-cause mortality $(n=155)$ (HR 1.74 (95\% Cl 1.20 to 2.52)) (both $\mathrm{p}<0.01)$. The association was independent of confounders of low MEEl $(<0.34 \mathrm{~mL} / \mathrm{s}$ per gram) identified in multivariable logistic regression analysis, including more severe AS, higher body mass index, lower LV midwall shortening and ejection fraction and presence of hypertension. Comparison of the Cox models with and without MEEi among the covariables demonstrated that MEEi significantly improved the prognostic yield (both $\mathrm{p}<0.01$ ).

Conclusions In patients with initially asymptomatic AS, low MEEi was associated with clustering of cardiometabolic risk factors, lower LV myocardial function and subsequent increased mortality during 4.3 years follow-up, independent of known prognosticators. Trial registration number NCT00092677.

\section{INTRODUCTION}

Data from the national echocardiographic database of Australia recently demonstrated that some patients with moderate aortic stenosis (AS) by conventional measures may have a long-term prognosis comparable with those with severe AS. ${ }^{1}$ This suggests that a more detailed risk classification is warranted to properly identify and manage high-risk patients with presumably non-severe AS. We

\section{Key questions}

What is already known about this subject?

- Myocardial energetic efficiency (MEEi) is an integrated marker of metabolic and left ventricular (LV) myocardial dysfunction that may be calculated by echocardiography as stroke volume per second per gram of LV mass. Low MEEi has been associated with impaired outcome in hypertensive and obese patients.

What does this study add?

- Among 1703 asymptomatic patients with aortic valve stenosis (AS) free from diabetes or known cardiovascular disease, low MEEi was associated with presence of cardiometabolic risk factors and LV myocardial dysfunction and identified a subgroup of AS patients with increased cardiovascular and allcause mortality.

How might this impact on clinical practice?

- Identification of low MEEi may help identifying a high-risk subgroup among asymptomatic AS patients free from diabetes and known cardiovascular disease.

have previously reported that comorbidities such as obesity and hypertension may contribute to impaired prognosis in nonsevere AS. ${ }^{23}$

In hypertension, a new subgroup of highrisk patients was recently identified, characterised by low myocardial energetic efficiency (MEEi). ${ }^{4}$ In previous reports, low MEEi has been associated with obesity or diabetes comorbidity, presence of subclinical LV myocardial dysfunction and impaired cardiovascular outcome. ${ }^{45}$ MEEi reflects the amount of blood ejected per second per gram of left ventricular (LV) mass. ${ }^{6}$ A low MEEi therefore represents inadequate systolic flow relative to LV mass. In AS, reduced flow is a powerful predictor of clinical outcomes, both in symptomatic ${ }^{7}$ and asymptomatic patients. ${ }^{8}$ MEEi is especially interesting in this regard as it also takes LV mass into account. ${ }^{23}$ Excessive LV 
mass has been associated with impaired prognosis both in asymptomatic non-severe and severe AS. ${ }^{9}{ }^{10}$ Whether low MEEi could identify a high-risk group among asymptomatic AS patients is not known. This was the aim of the present analysis.

\section{METHODS}

\section{Patient population}

The present post hoc analysis was performed within the prospective Simvastatin and Ezetimibe in Aortic Stenosis (SEAS) study that randomised 1873 asymptomatic patients with AS, defined as aortic valve thickening and peak aortic jet velocity between $\geq 2.5$ and $\leq 4.0 \mathrm{~m} / \mathrm{s}$, to double-blinded, placebo-controlled combined treatment with simvastatin $40 \mathrm{mg}$ and ezetimibe $10 \mathrm{mg}$ daily for a median of 4.3 years. ${ }^{11}$

Patients with coronary, cerebral or peripheral vascular disease, diabetes mellitus, other significant valvular heart disease, systolic heart failure (ejection fraction $<40 \%$ ), renal insufficiency or patients with any indication or contraindication to lipid-lowering therapy were not included in the SEAS study. ${ }^{11}$ The core laboratory received baseline echocardiograms in 1788 patients, and MEEi could be calculated in 1703 (95.2\%). Compared with the 85 patients without MEEi measurements, the present study population did not differ in age, sex distribution or body mass index (all $\mathrm{p}>0.3$ ). Obesity was defined as body mass index $\geq 30 \mathrm{~kg} / \mathrm{m}^{2}{ }^{2}$ Hypertension was defined as history of hypertension or use of antihypertensive treatment reported by the attending physician or elevated blood pressure at the baseline clinical visit. ${ }^{11}$ There was no patient or public involvement in the design or analysis of this study. All patients gave written informed consent to participate in the SEAS study.

\section{Echocardiography}

Baseline echocardiograms were obtained at 173 study centres in seven European countries (Norway, Sweden, Finland, Denmark, UK, Ireland and Germany) following a standardised protocol. ${ }^{911}$ All echocardiograms were sent for expert interpretation at the SEAS echocardiography core laboratory in Bergen, Norway. The SEAS echocardiography protocol and methods have been published previously. ${ }^{911}$ Quantitative echocardiography was performed following the joint European Association of Cardiovascular Imaging and American Society of Echocardiography recommendations. ${ }^{12} \mathrm{LV}$ mass was measured by an autopsy-validated method and indexed to body height in the allometric power of 2.7. ${ }^{13} \mathrm{LV}$ hypertrophy was identified by the prognostically validated cutoff values $\mathrm{LV}$ mass $/$ height ${ }^{2.7} \geq 46.7 \mathrm{~g} / \mathrm{m}^{2.7}$ in women and $49.2 \mathrm{~g} / \mathrm{m}^{2.7}$ in men. ${ }^{9}$ Stroke volume was calculated by Doppler and indexed for body surface area and considered low if $\leq 35 \mathrm{~mL} / \mathrm{m}^{2}{ }^{14}$ MEEi is estimated as the ratio of stroke work to estimated myocardial oxygen consumption. In this equation, stroke work is calculated as the product of the systolic blood pressure and the stroke volume by echocardiography. Since myocardial oxygen consumption is not limited to the systole, the time used is the cardiac cycle. To have an expression of myocardial mechanical efficiency per $1 \mathrm{~s}$ in the individual patient, heart rate (beats per min) was divided by 60 . MEE was calculated by the equation: stroke volume/([heart rate/60]) and indexed to LV mass (MEEi). ${ }^{4}$

Peak aortic jet velocity was measured from different windows by imaging and non-imaging transducers, and the highest velocity was used for tracing of the timevelocity integral. The aortic valve area was calculated by the continuity equation using velocity time integrals. Pressure recovery adjusted valve area (energy loss), valvuloarterial impedance and LV midwall shortening were calculated using previously validated formulas. ${ }^{15-17}$

\section{Study end-points}

Prespecified study end-points were adjudicated by an independent committee. ${ }^{11}$ The present analysis targeted heart failure hospitalisation, cardiovascular death, allcause mortality and the composite endpoint heart failure hospitalisation and cardiovascular death. ${ }^{11}$

\section{Statistical analysis}

Data management and analysis was performed using IBM SPSS V.26.0 software (IBM, Armonk, New York, USA) and R V.3.6.1 (The R foundation for Statistical Computing, Vienna, Austria) with the packages within Tidyverse, Survival and Greg. From univariable Cox regression, generalised additive models with smoothing splines were used to visualise the unadjusted continuous effect of MEEi on the study end-points heart failure hospitalisation, cardiovascular death and all-cause mortality. Increased hazard was identified if MEEi $<0.34 \mathrm{~mL} / \mathrm{s}$ per gram for all end-points. Consequently, the study cohort was divided into MEEi $<0.34 \mathrm{~mL} / \mathrm{s}$ per gram (low MEEi), corresponding to the upper limit of the lower tertile in the cohort, and the remaining cohort. Continuous variables are presented as mean $\pm \mathrm{SD}$ and categorical variables as percentages. Groups were compared by Student's unpaired t-tests or $\chi^{2}$ test, as appropriate. To test survival in patients with low MEEi in different degrees of AS severity, the study population was grouped into mild (aortic jet velocity $<3.0 \mathrm{~m} / \mathrm{s}$ ), moderate (aortic jet velocity $3.0-3.9 \mathrm{~m} / \mathrm{s}$ ) and severe AS (aortic jet velocity $\geq 4.0 \mathrm{~m} / \mathrm{s}$ ). Cumulative event rates were calculated by Kaplan-Meier and compared between groups using log-rank test. Independent covariables of low MEEi were identified in multivariable logistic regression analysis. Outcome was assessed in Cox regression analyses and reported as $\mathrm{HR}$ and $95 \%$ CI. The multivariable Cox models were adjusted for covariables of low MEEi including sex, age $>65$ years, hypertension, body mass index, low glomerular filtration rate, LV ejection fraction, midwall shortening, aortic valve area and randomised study treatment. The contribution of low MEEi to the model yield was tested by comparing the Aikaike information criterion (AIC) for the adjusted Cox model with and without low MEEi among the covariables 

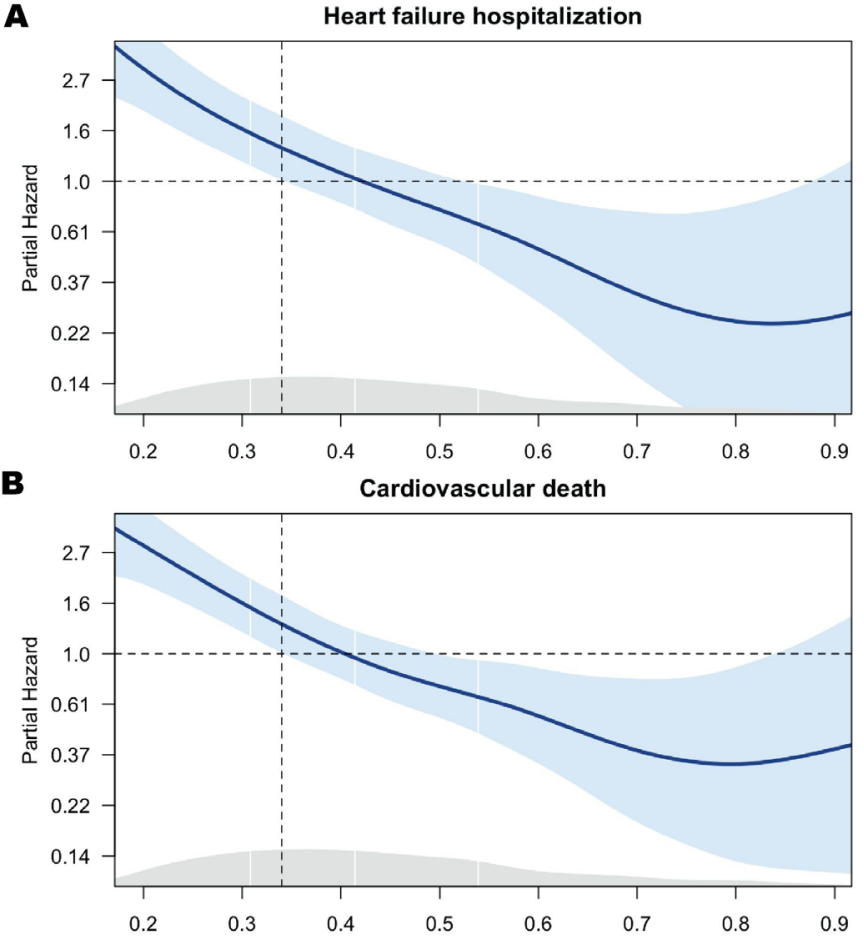

C

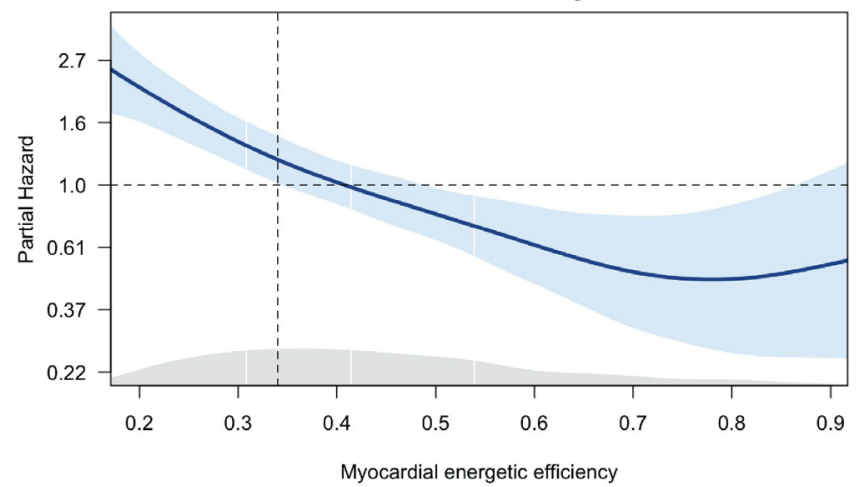

Figure 1 Univariable relation between the continuous effect of MEEi on heart failure hospitalisation (A), cardiovascular death (B) and all-cause mortality (C) using generalised additive models. The solid lines display the observed association and the shaded areas the 95\% Cls. Crossed dotted lines represent the crosspoint, that is, the point where the $95 \% \mathrm{Cl}$ crosses the partial hazard of 1 .

by the likelihood ratio test. In a second set of these Cox models, low MEEi was replaced by MEEi as a continuous variable. Receiver-operating characteristic curve analysis was used to compare prediction of combined cardiovascular death and heart failure hospitalisation by MEEi versus all composites of MEEi entered simultaneously. A $p$ value $<0.05$ was regarded statistically significant in all analyses.

\section{RESULTS}

\section{Prevalence and covariables of low MEEi at baseline}

In generalised additive models, an MEEi $<0.34 \mathrm{~mL} / \mathrm{s}$ per gram was identified as a threshold value for increased risk for heart failure hospitalisation, cardiovascular death and
Table 1 Clinical characteristics of patients with and without low MEE

\begin{tabular}{|c|c|c|c|}
\hline Variables & $\begin{array}{l}\text { Low MEEi } \\
\mathrm{n}=569\end{array}$ & $\begin{array}{l}\text { No low } \\
\text { MEEi } \\
n=1134\end{array}$ & P value \\
\hline Age (years) & $68 \pm 10$ & $67 \pm 10$ & 0.016 \\
\hline Age above 65 years (\%) & 66 & 64 & 0.319 \\
\hline Women (\%) & 35 & 41 & 0.008 \\
\hline Systolic blood pressure (mm Hg) & $149 \pm 20$ & $147 \pm 21$ & 0.013 \\
\hline Diastolic blood pressure $(\mathrm{mm} \mathrm{Hg})$ & $84 \pm 11$ & $82 \pm 10$ & $<0.001$ \\
\hline Heart rate (beats/min) & $72 \pm 12$ & $63 \pm 10$ & $<0.001$ \\
\hline Hypertension (\%) & 88 & 81 & $<0.001$ \\
\hline Body surface area $\left(\mathrm{cm}^{2}\right)$ & $1.93 \pm 0.20$ & $1.88 \pm 0.19$ & $<0.001$ \\
\hline Body mass index $\left(\mathrm{kg} / \mathrm{m}^{2}\right)$ & $27.9 \pm 4.7$ & $26.3 \pm 4.1$ & $<0.001$ \\
\hline Obesity (\%) & 28 & 16 & $<0.001$ \\
\hline $\begin{array}{l}\text { Estimated glomerular filtration rate } \\
\left(\mathrm{mL} / \mathrm{min} / 1.73 \mathrm{~m}^{2}\right)\end{array}$ & $83.8 \pm 16.7$ & $85.7 \pm 17.3$ & 0.039 \\
\hline Low glomerular filtration rate & $7.0 \%$ & $7.4 \%$ & 0.797 \\
\hline Antihypertensive drugs (n) & $1.3 \pm 1.2$ & $1.2 \pm 1.2$ & 0.006 \\
\hline
\end{tabular}

MEEi, myocardial energetic efficiency index.

all-cause mortality (figure 1 ). Patients with MEEi $<0.34$ $\mathrm{mL} / \mathrm{s}$ per gram (low MEEi) were older, more often men, obese and hypertensive, with higher blood pressure and heart rate, and lower renal function compared with patients without low MEEi (all $\mathrm{p}<0.05$, table 1 ).

Patients with low MEEi had higher prevalence of LV hypertrophy and lower LV systolic function measured by ejection fraction and midwall shortening (all $\mathrm{p}<0.05$, table 2). Patients with low MEEi also had more severe AS (table 2).

In multivariable logistic regression analysis, low MEEi was independently associated with prevalent hypertension, more severe AS, higher body mass index and lower midwall shortening (all $\mathrm{p}<0.05$, table 3 ).

\section{Association of low MEEi at study baseline with outcome}

During a median follow-up of 4.3 years, 66 heart failure hospitalisations, 80 cardiovascular deaths and 155 deaths from any cause occurred in the total study cohort. Presence of low MEEi was associated with a 2.0-fold higher HR for all-cause mortality and a 2.8-fold higher HR for both heart failure hospitalisation and cardiovascular death in univariable Cox regression (all p<0.001, table 4). In multivariable analyses, the association of low MEEi with higher cardiovascular death and all-cause mortality remained significant (both $\mathrm{p}<0.05$, table 4 ), while the association with heart failure hospitalisation did not (table 4). In a second set of Cox models, replacing low MEEi by MEEi as a continuous variable, lower MEEi was significantly associated with higher cardiovascular and all-cause mortality (both $\mathrm{p}<0.001$ ) but not with heart failure hospitalisation $(\mathrm{p}=0.051)$ (table 5). When grouping patients by AS severity based on peak aortic jet velocity, low MEEi was 


\begin{tabular}{|c|c|c|c|}
\hline Variables & Low MEEi $n=569$ & No low MEEi $n=1134$ & $P$ value \\
\hline \multicolumn{4}{|l|}{ Aortic valve } \\
\hline Aortic annulus diameter (cm) & $2.13 \pm 0.27$ & $2.22 \pm 0.25$ & $<0.001$ \\
\hline Peak aortic jet velocity (m/s) & $3.1 \pm 0.6$ & $3.1 \pm 0.5$ & 0.235 \\
\hline Mean aortic gradient (mm Hg) & $24 \pm 9$ & $22 \pm 8$ & 0.012 \\
\hline Aortic valve area $\left(\mathrm{cm}^{2}\right)$ & $1.10 \pm 0.40$ & $1.36 \pm 0.47$ & $<0.001$ \\
\hline Energy loss $\left(\mathrm{cm}^{2}\right)$ & $1.38 \pm 0.64$ & $1.85 \pm 0.93$ & $<0.001$ \\
\hline \multicolumn{4}{|l|}{ Left ventricle } \\
\hline LV end-diastolic diameter (cm) & $5.20 \pm 0.65$ & $4.95 \pm 0.61$ & $<0.001$ \\
\hline LV end-systolic diameter (cm) & $3.38 \pm 0.59$ & $3.10 \pm 0.52$ & $<0.001$ \\
\hline LV mass $(\mathrm{g})$ & $238 \pm 77$ & $173 \pm 51$ & $<0.001$ \\
\hline LV hypertrophy (\%) & 61 & 22 & $<0.001$ \\
\hline Ejection fraction $(\%)$ & $65 \pm 7$ & $67 \pm 6$ & $<0.001$ \\
\hline Stroke volume (mL) & $71 \pm 20$ & $92 \pm 25$ & $<0.001$ \\
\hline Low stroke volume index $\left(\leq 35 \mathrm{~mL} / \mathrm{m}^{2}\right)(\%)$ & 47.6 & 9.9 & $<0.001$ \\
\hline Midwall shortening (\%) & $15.6 \pm 3.0$ & $17.7 \pm 3.2$ & $<0.001$ \\
\hline Valvuloarterial impedance $\left(\mathrm{mm} \mathrm{Hg} / \mathrm{mL} / \mathrm{m}^{2}\right)$ & $4.8 \pm 1.2$ & $3.5 \pm 1.0$ & $<0.001$ \\
\hline MEEi (mL/s per gram) & $0.26 \pm 0.06$ & $0.54 \pm 0.16$ & \\
\hline
\end{tabular}

LV, left ventricular; MEEi, myocardial energetic efficiency index.

associated with reduced survival in patients with mild and moderate AS but not in patients with severe AS (figure 2).

We tested whether low MEEi added to the yield of these multivariable Cox models by comparing the models with and without low MEEi included. The inclusion of low MEEi significantly improved model prediction of the endpoint of all-cause mortality (AIC 1987.9 vs 1981.4, $\mathrm{p}<0.01$ ) and cardiovascular death (AIC 1025.6 vs 1015.3, $\mathrm{p}<0.001$ ) but not for heart failure hospitalisation (AIC 855.3 vs $853.8, \mathrm{p}=0.059)$.

In C-statistics, neither MEEi as a continuous variable nor low MEEi significantly improved identification of patients at increased risk for the composite endpoint heart failure hospitalisation or cardiovascular death compared with

\begin{tabular}{|c|c|c|c|}
\hline Variables & OR & $95 \% \mathrm{Cl}$ & $P$ value \\
\hline Male sex & 2.65 & 1.92 to 3.66 & 0.087 \\
\hline Age $>65$ years & 1.09 & 0.81 to 1.47 & 0.555 \\
\hline Low GFR & 1.01 & 1.00 to 1.02 & 0.134 \\
\hline Hypertension & 1.50 & 1.06 to 2.14 & 0.023 \\
\hline Body mass index $\left(\mathrm{kg} / \mathrm{m}^{2}\right)$ & 1.11 & 1.08 to 1.15 & $<0.001$ \\
\hline Midwall shortening (\%) & 0.83 & 0.80 to 0.87 & $<0.001$ \\
\hline Ejection fraction (\%) & 0.98 & 0.97 to 1.00 & 0.087 \\
\hline Aortic valve area $\left(\mathrm{cm}^{2}\right)$ & 0.13 & 0.09 to 0.18 & $<0.001$ \\
\hline
\end{tabular}

GFR, glomerular filtration rate; MEEi, myocardial energetic efficiency. the MEEi components LV mass, stroke volume and heart rate when these were entered simultaneously.

\section{DISCUSSION}

This study is the first to demonstrate that presence of low LV MEEi is associated with increased mortality in asymptomatic AS patients free from diabetes and known cardiovascular and renal disease. This association was independent of the well-known clinical prognostic factors in AS that were also identified as covariables of low MEEi, including male sex ${ }^{18}$ hypertension, ${ }^{3}$ higher body mass index,${ }^{2}$ lower LV midwall shortening ${ }^{19}$ and ejection fraction, ${ }^{20}$ and more severe AS. ${ }^{15} 2122$ Interestingly, the association with increased mortality was found in asymptomatic patients with mild or moderate AS, but not among those with classical severe AS identified by a peak jet velocity $\geq 4.0 \mathrm{~m} / \mathrm{s}$, which comprised a small number of patients. The results of the present study expand recent observations from the Strong Heart Study, demonstrating low MEEi defined as $\leq 0.34 \mathrm{~mL} / \mathrm{s}$ per gram as a predictor of incident heart failure in 1912 North-American Indians without prevalent cardiovascular disease and with normal LV ejection fraction. ${ }^{5}$ Also in the Campania Salute Network project, a prospective follow-up registry including more than 12000 patients with hypertension, low MEEi predicted increased risk for major cardiovascular events, including hospitalisation for myocardial infarction, stroke, heart failure, coronary revascularisation, angina pectoris and atrial fibrillation, as well as sudden cardiac death. ${ }^{4}$ However, in that study low MEEi 
Table 4 Association of low MEEi with outcome

\begin{tabular}{|c|c|c|c|c|c|c|}
\hline \multirow[b]{2}{*}{ Variables } & \multicolumn{2}{|c|}{ HF hospitalisation $(n=66)$} & \multicolumn{2}{|l|}{ CV death $(n=80)$} & \multicolumn{2}{|c|}{ All-cause mortality $(n=155)$} \\
\hline & HR $(95 \% \mathrm{Cl})$ & $P$ value & HR (95\% Cl) & $P$ value & HR $(95 \% \mathrm{Cl})$ & $P$ value \\
\hline \multicolumn{7}{|l|}{ Univariable analysis } \\
\hline \multicolumn{7}{|l|}{ Multivariable analysis } \\
\hline Low MEEi $(<0.34 \mathrm{~mL} / \mathrm{s}$ per gram) & $1.73(0.97$ to 3.07$)$ & 0.062 & 2.53 (1.50 to 4.28$)$ & 0.001 & 1.74 (1.20 to 2.52$)$ & 0.003 \\
\hline Aortic valve area $\left(\mathrm{cm}^{2}\right)$ & $0.26(0.12$ to 0.58$)$ & 0.001 & 1.15 (0.64 to 2.07$)$ & 0.642 & $0.88(0.57$ to 1.35$)$ & 0.541 \\
\hline Body mass index $\left(\mathrm{kg} / \mathrm{m}^{2}\right)$ & 1.04 (0.98 to 1.10$)$ & 0.171 & 0.95 (0.90 to 1.01$)$ & 0.099 & 0.98 (0.94 to 1.02$)$ & 0.354 \\
\hline Ejection fraction (\%) & $0.93(0.90$ to 0.96$)$ & $<0.001$ & 0.98 (0.95 to 1.01$)$ & 0.166 & $0.99(0.96$ to 1.01$)$ & 0.251 \\
\hline Midwall shortening (\%) & 0.96 (0.88 to 1.04$)$ & 0.296 & $0.93(0.87$ to 1.01$)$ & 0.101 & $0.95(0.90$ to 1.01$)$ & 0.100 \\
\hline Low GFR & $0.74(0.23$ to 2.39$)$ & 0.619 & $0.41(0.10$ to 1.68$)$ & 0.215 & $0.83(0.41$ to 1.70$)$ & 0.607 \\
\hline
\end{tabular}

CV, cardiovascular; GFR, glomerular filtration rate; HF, heart failure; MEEi, myocardial energetic efficiency index.

was identified as $<0.29 \mathrm{~mL} / \mathrm{s} / \mathrm{g}$, reflecting the lower quartile of MEEi in their cohort. ${ }^{4}$ Adding to these studies, the cut-off value defining low MEEi in our analysis was identified in generalised additive models of the association of MEEi as a continuous variable with outcomes. Of note, the same threshold value for increased risk was found for heart failure hospitalisation, cardiovascular death and allcause mortality.

The high-risk phenotype associated with low MEEi in the present study was similar to that identified in these previous studies, including presence of higher LV mass, LV myocardial dysfunction, dyslipidaemia, cardiovascular inflammation, hypertension and obesity. ${ }^{45}$ Arterial hypertension is a common comorbidity in AS patients. ${ }^{323}$ In a large cohort study of 5.4 million subjects without known cardiovascular disease in the UK, presence of hypertension increased the risk of AS development. ${ }^{24}$ In AS, hypertension has been associated with increased valvuloarterial impedance, ${ }^{25}$ lower arterial compliance ${ }^{26}$ and higher mortality. ${ }^{3}$ Importantly, although hypertension was associated with lower MEEi, low MEEi predicted increased mortality independent of hypertension in the present study. Treating hypertension in patients with mild and moderate AS may increase MEEi and reduce mortality in these patients. A recent consensus document on management of hypertension in AS by the European Society of Cardiology provided guidance for antihypertensive treatment in AS patients, recommending ACE inhibitors or angiotensin receptor blockers as first-line therapy. ${ }^{27}$

Low stroke volume index has been associated with increased risk of heart failure hospitalisations and mortality in AS. ${ }^{78}$ Presence of lower stroke volume, higher heart rate and higher LV mass are known determinants of higher risk in AS, so it is not surprising that combining them is also prognostically significant. However, stroke volume is delivered during ejection. In contrast, MEEi reflects the amount of blood pumped by per each gram of LV mass per second. Although MEEi is calculated as the ratio of stroke volume and heart rate indexed to LV mass, low stroke volume index was found in less than $50 \%$ of patients with low MEEi. Thus, low MEEi does not merely reflect a low flow state but should rather be interpreted as a marker of metabolic heart disease, incorporating presence of metabolic disorders like obesity, hypertension and insulin resistance with LV myocardial dysfunction. ${ }^{5}$

Also obesity is common in patients with AS and associated with increased all-cause mortality independent of the severity of AS. ${ }^{2}$ Obesity also leads to overestimation

Table 5 Association of MEEi as a continuous variable with outcome

\begin{tabular}{|c|c|c|c|c|c|c|}
\hline \multirow[b]{2}{*}{ Variables } & \multicolumn{2}{|c|}{ HF hospitalisation $(n=66)$} & \multicolumn{2}{|l|}{ CV death $(n=80)$} & \multicolumn{2}{|c|}{ All-cause mortality $(n=155)$} \\
\hline & HR (95\% Cl) & P value & HR $(95 \% \mathrm{Cl})$ & P value & HR (95\% Cl) & P value \\
\hline \multicolumn{7}{|l|}{ Univariable analysis } \\
\hline \multicolumn{7}{|l|}{ Multivariable analysis } \\
\hline MEEi (mL/s per gram) & $0.11(0.01$ to 1.01$)$ & 0.051 & $0.03(0.01$ to 0.19$)$ & $<0.001$ & $0.15(0.04$ to 0.53$)$ & 0.004 \\
\hline
\end{tabular}

CV, cardiovascular; HF, heart failure; MEEi, myocardial energetic efficiency index. 

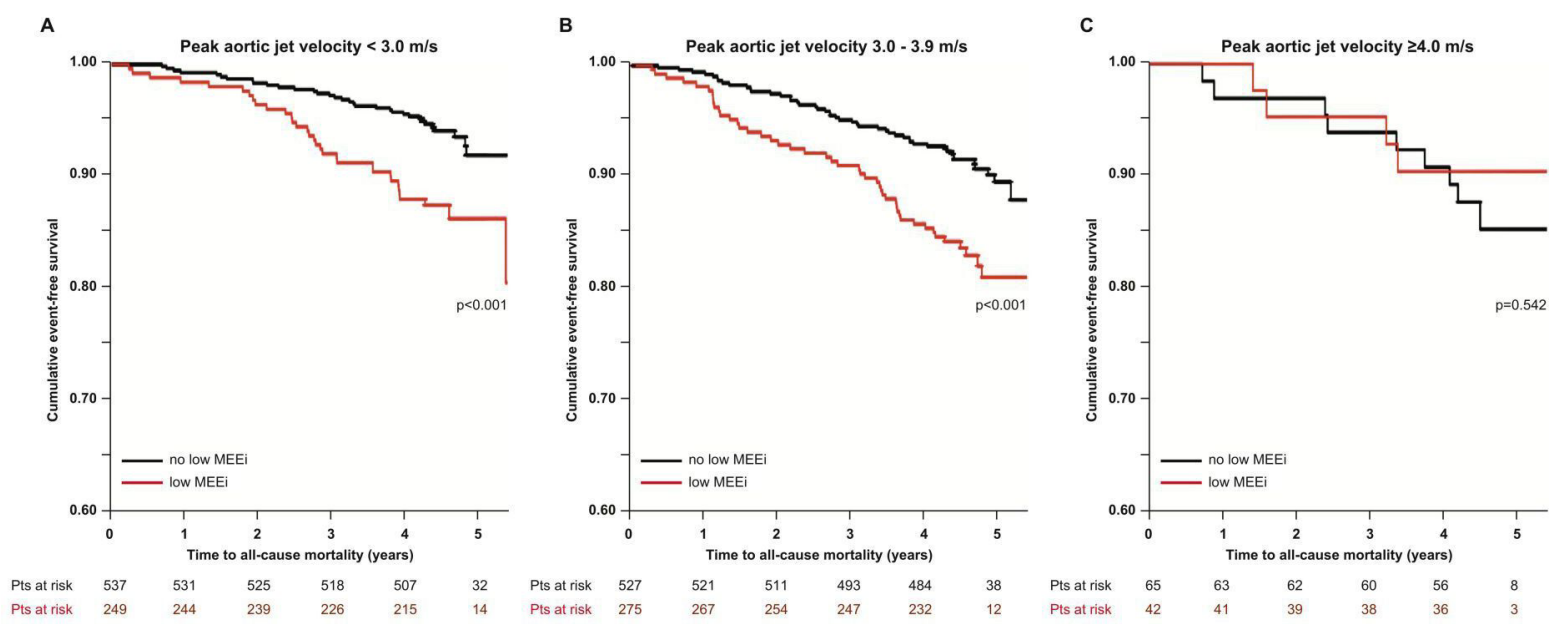

Figure 2 Kaplan-Meier plot of overall survival in patients with low MEEi compared with normal MEEi in patients with peak aortic jet velocity $<3.0 \mathrm{~m} / \mathrm{s}(\mathrm{A}), 3.0-3.9 \mathrm{~m} / \mathrm{s}(\mathrm{B})$ and $\geq 4.0 \mathrm{~m} / \mathrm{s}(\mathrm{C})$. MEEi, myocardial energetic efficiency.

of true AS severity and underestimation of flow when based on aortic valve area and stroke volume indexed to body surface area, respectively. ${ }^{8}$ Overestimation of true AS severity implies the risk of premature referral for aortic valve replacement without favourable influence on survival.

As demonstrated, low MEEi improved prediction of cardiovascular and all-cause mortality independent of hypertension, older age, higher body mass index and lower LV systolic function and AS severity in the present study. However, many of the deaths in the low MEEi group could probably not have been prevented by aortic valve replacement since they are primarily related to comorbidity-associated risk including arterial hypertension or obesity. This points to the need for better cardiovascular risk factor management in AS to reduce mortality. ${ }^{23}$

It is well demonstrated that global longitudinal strain is a better marker of early LV dysfunction with superior prognostic value compared with LV ejection fraction in predicting major adverse cardiac events. ${ }^{28}$ In fact, LV ejection fraction has limited prognostic value when in the low-normal range or higher. ${ }^{29}$ During progression of AS, LV global systolic myocardial function declines, whether measured by midwall shortening or global LV longitudinal strain. ${ }^{25}{ }^{30}$ Both these variables have also documented prognostic value in AS. ${ }^{19} 31$ In hypertension, it has been documented that midwall shortening and global longitudinal strain have rather similar performance in detecting subclinical myocardial dysfunction. ${ }^{32}$ However, in the present analysis, MEEi predicted higher mortality independent of midwall shortening. Whether MEEi identifies LV myocardial dysfunction comparable with global longitudinal strain could not be tested in the present study since data on global longitudinal strain was not available in the dataset. However, Mancusi $e t a l^{33}$ recently demonstrated in a study of 480 obese subjects that both global longitudinal strain and midwall shortening decreased in parallel with lower MEEi.

\section{Study limitations}

The SEAS study excluded patients with atherosclerotic disease, diabetes and LV ejection fraction $<40 \%$ by design, and the implementation of the current results in less selective groups of AS patients should be done with caution. Furthermore, positron emission tomography, the gold standard method for assessment of MEEi, ${ }^{34}$ was not performed in the SEAS study. However, echocardiography is the recommended method in AS evaluation, ${ }^{14}$ and MEEi by echocardiography has been prognostically validated in non-AS cohorts. ${ }^{45}$ Finally, data on global longitudinal strain, right ventricular function and B-type natriuretic peptides were not available in the SEAS study. Of note, baseline echocardiograms were obtained in 2002-2004 and mostly submitted to the echocardiography core laboratory on video home system videotapes. Future studies should determine whether MEEi may provide additional prognostic information beyond global longitudinal strain, right ventricular function measures and B-type natriuretic peptides in patients with asymptomatic mild to moderate AS. Furthermore, additional studies are particularly needed to explore the impact of cardiovascular risk factor management in AS on MEEi and outcome and to further validate cut-off values for low MEEi that can be used in clinical algorithms for risk assessment is asymptomatic AS patients.

\section{CONCLUSION}

Low MEEi is characterised by presence of metabolic risk factors and LV myocardial dysfunction and is associated with increased mortality in patients with asymptomatic mild to moderate AS free from diabetes and known cardiovascular disease. Identification of low MEEi may help identifying a subgroup of asymptomatic AS patients with metabolic myocardial dysfunction and reduced survival that may particularly benefit from thorough cardiovascular risk factor management. 
Contributors EB, EE, DC, HM, CM, AR and EG had designed the study and were responsible for data collection. EB and EG did the statistical analysis and prepared the manuscript. All authors contributed to reviewing or revising the manuscript and approved the final version. EG is responsible for the overall content as guarantor.

Funding The Simvastatin and Ezetimibe in Aortic Stenosis study was sponsored by MSP Singapore Company, LLC, Singapore, a partnership between Merck \& Co, Inc, and the Schering-Plough Corporation.

Competing interests None declared.

Patient consent for publication Not required.

Ethics approval The SEAS study was approved by regional ethics committees in all participating countries.

Provenance and peer review Not commissioned; externally peer reviewed.

Data availability statement Data are available on reasonable request. All data relevant to the study are included in the article or uploaded as supplementary information

Open access This is an open access article distributed in accordance with the Creative Commons Attribution Non Commercial (CC BY-NC 4.0) license, which permits others to distribute, remix, adapt, build upon this work non-commercially, and license their derivative works on different terms, provided the original work is properly cited, appropriate credit is given, any changes made indicated, and the use is non-commercial. See: http://creativecommons.org/licenses/by-nc/4.0/.

\section{ORCID iDs}

Edda Bahlmann http://orcid.org/0000-0002-4242-0535

Eigir Einarsen http://orcid.org/0000-0002-6672-3370

\section{REFERENCES}

1 Strange G, Stewart S, Celermajer D, et al. Poor long-term survival in patients with moderate aortic stenosis. J Am Coll Cardiol 2019;74:1851-63.

2 Rogge BP, Cramariuc D, Lønnebakken MT, et al. Effect of overweight and obesity on cardiovascular events in asymptomatic aortic stenosis: a seas substudy (simvastatin ezetimibe in aortic stenosis). J Am Coll Cardiol 2013;62:1683-90.

3 Rieck Åshild E, Cramariuc D, Boman K, et al. Hypertension in aortic stenosis: implications for left ventricular structure and cardiovascular events. Hypertension 2012;60:90-7.

4 de Simone G, Izzo R, Losi MA, et al. Depressed myocardial energetic efficiency is associated with increased cardiovascular risk in hypertensive left ventricular hypertrophy. $J$ Hypertens 2016;34:1846-53.

5 Losi M-A, Izzo R, Mancusi C, et al. Depressed myocardial energetic efficiency increases risk of incident heart failure: the strong heart study. J Clin Med 2019;8:1044.

6 de Simone G, Chinali M, Galderisi M, et al. Myocardial mechanoenergetic efficiency in hypertensive adults. J Hypertens 2009;27:650-5.

7 Eleid MF, Sorajja P, Michelena HI, et al. Flow-gradient patterns in severe aortic stenosis with preserved ejection fraction: clinical characteristics and predictors of survival. Circulation 2013;128:1781-9.

8 Lønnebakken MT, De Simone G, Saeed S, et al. Impact of stroke volume on cardiovascular risk during progression of aortic valve stenosis. Heart 2017;103:1443-8.

9 Gerdts E, Rossebø AB, Pedersen TR, et al. Relation of left ventricular mass to prognosis in initially asymptomatic mild to moderate aortic valve stenosis. Circ Cardiovasc Imaging 2015;8:e003644

10 Cioffi G, Faggiano P, Vizzardi E, et al. Prognostic effect of inappropriately high left ventricular mass in asymptomatic severe aortic stenosis. Heart 2011;97:301-7.

11 Rossebø AB, Pedersen TR, Boman K, et al. Intensive lipid lowering with simvastatin and ezetimibe in aortic stenosis. $N$ Engl $\mathrm{J}$ Med 2008;359:1343-56.

12 Lang RM, Badano LP, Mor-Avi V, et al. Recommendations for cardiac chamber quantification by echocardiography in adults: an update from the American Society of Echocardiography and the European Association of Cardiovascular Imaging. Eur Heart J Cardiovasc Imaging 2015;16:233-71.
13 Devereux RB, Alonso DR, Lutas EM, et al. Echocardiographic assessment of left ventricular hypertrophy: comparison to necropsy findings. Am J Cardiol 1986;57:450-8.

14 Nishimura RA, Otto CM, Bonow RO, et al. 2014 AHA/ACC guideline for the management of patients with valvular heart disease: a report of the American College of Cardiology/American Heart Association task force on practice guidelines. Circulation 2014;129:e521-643.

15 Bahlmann E, Gerdts E, Cramariuc D, et al. Prognostic value of energy loss index in asymptomatic aortic stenosis. Circulation 2013;127:1149-56.

16 Briand M, Dumesnil JG, Kadem L, et al. Reduced systemic arterial compliance impacts significantly on left ventricular afterload and function in aortic stenosis: implications for diagnosis and treatment. J Am Coll Cardiol 2005;46:291-8.

17 de Simone G, Devereux RB, Roman MJ, et al. Assessment of left ventricular function by the midwall fractional shortening/endsystolic stress relation in human hypertension. J Am Coll Cardiol 1994;23:1444-51.

18 Cramariuc D, Rogge BP, Lønnebakken MT, et al. Sex differences in cardiovascular outcome during progression of aortic valve stenosis. Heart 2015;101:209-14

19 Cramariuc D, Bahlmann E, Egstrup K, et al. Prognostic impact of impaired left ventricular midwall function during progression of aortic stenosis. Echocardiography 2021;38:31-8.

20 Capoulade R, Le Ven F, Clavel M-A, et al. Echocardiographic predictors of outcomes in adults with aortic stenosis. Heart 2016;102:934-42.

21 Rosenhek R, Klaar U, Schemper M, et al. Mild and moderate aortic stenosis. natural history and risk stratification by echocardiography. Eur Heart J 2004;25:199-205.

22 Pellikka PA, Sarano ME, Nishimura RA, et al. Outcome of 622 adults with asymptomatic, hemodynamically significant aortic stenosis during prolonged follow-up. Circulation 2005;111:3290-5.

23 Mancusi C, de Simone G, Hitij JB. Management of patients with combined arterial hypertension and aortic valve stenosis: a consensus document from the Council on hypertension and Council on valvular heart disease of the European Society of cardiology, the European association of cardiovascular imaging (EACVI), the European association of percutaneous cardiovascular interventions (EAPCI). Eur Heart J Cardiovasc Pharmacother 2020;7.

24 Rahimi K, Mohseni H, Kiran A, et al. Elevated blood pressure and risk of aortic valve disease: a cohort analysis of 5.4 million UK adults. Eur Heart J 2018;39:3596-603.

25 Cramariuc D, Cioffi G, Rieck AE, et al. Low-flow aortic stenosis in asymptomatic patients: valvular-arterial impedance and systolic function from the seas substudy. JACC Cardiovasc Imaging 2009;2:390-9.

26 Bahlmann E, Cramariuc D, Saeed S, Lønnebakken MT, et al. Low systemic arterial compliance is associated with increased cardiovascular morbidity and mortality in aortic valve stenosis. Heart 2019;105:1507-14

27 Lindman BR, Otto CM. Time to treat hypertension in patients with aortic stenosis. Circulation 2013;128:1281-3.

28 Kalam K, Otahal P, Marwick TH. Prognostic implications of global LV dysfunction: a systematic review and meta-analysis of global longitudinal strain and ejection fraction. Heart 2014;100:1673-80.

29 Solomon SD, Anavekar N, Skali H, et al. Influence of ejection fraction on cardiovascular outcomes in a broad spectrum of heart failure patients. Circulation 2005;112:3738-44

30 Cramariuc D, Gerdts E, Davidsen ES, et al. Myocardial deformation in aortic valve stenosis: relation to left ventricular geometry. Heart 2010;96:106-12

31 Vollema EM, Sugimoto T, Shen M, et al. Association of left ventricula global longitudinal strain with asymptomatic severe aortic stenosis: natural course and prognostic value. JAMA Cardiol 2018;3:839-47.

32 Lembo M, Santoro C, Sorrentino R, et al. Interrelation between midwall mechanics and longitudinal strain in newly diagnosed and never-treated hypertensive patients without clinically defined hypertrophy. J Hypertens 2020;38:295-302.

33 Mancusi C, Midtbø $\mathrm{H}$, De Luca N, et al. Association of myocardial energetic efficiency with circumferential and longitudinal left ventricular myocardial function in subjects with increased body mass index (the FATCOR study). J Clin Med 2021;10:1581.

34 Hansson NHS, Sörensen J, Harms HJ, et al. Myocardial oxygen consumption and efficiency in aortic valve stenosis patients with and without heart failure. J Am Heart Assoc 2017;6:e004810. 